\section{Vitamin K-deficient Mutants of Bacteria}

AuxотRорніс mutants of bacteria with various metabolic blocks have proved useful for the elucidation of intermediary steps in biosynthetic pathways. ${ }^{1}$. Until recently this approach has not been applied to the biosynthesis of $\mathrm{K}$ vitamins (menaquinones), chiefly because of the lack of adequate vitamin $K$-deficient mutants ${ }^{2}$ which would accumulate significant intermediary metabolites. We have isolated some menaphthone-requiring, small-colony mutants of Staphylococcus aureus by selection with neomycin ${ }^{3}$ and found that they grow and respire normally on media supplemented with menadione. This could be attributed to bridging of the main electron transport chain by menadione, which is known to be capable of by-passing flavin, vitamin K2 and cytochrome $b$ (ref. 4). If so, the mutants stimulated by menadione might equally represent flavin-deficient, vitamin K-deficient, or eytochrome $b$ deficient mutants. Alternatively, if menadione is an intermediate in the biosynthesis of $K$ vitamins, these mutants may simply be of the vitamin $K$-deficient type. The biosynthesis of menaquinones up to chorismic acid follows the common pathway of aromatic amino-acids ${ }^{2}$, so that some of the vitamin $\mathrm{K}$-deficient mutants stimulated by menadione might be stimulated by shikimic acid as well, provided that the latter can penctrate the bacterial cell.

Here we present results which seem to confirm the second hypothesis concerning the mutants stimulated by menadione. A number of these mutants were stimulated by shikimic acid, indicating a metabolic block before synthesis of the latter. Thin-layer chromatography of the lipidic extract of one such mutant (SHSL1) grown on nutrient agar without shikimic acid lacked menaquinones, but when the medium was supplemented with $300 \mu \mathrm{g} / \mathrm{ml}$. shikimic acid the mutant was able to synthesize vitamin K2. When the same mutant was grown in the presence of menadione (l $\mu \mathrm{g} / \mathrm{ml}$.) the lipidic extract contained vitamin K2; we conclude therefore that menadione can represent an intermediary metabolite in the biosynthesis of vitamin $\mathrm{K} 2$, or can be readily converted into one. The alternative hypothesis concerning menadione-involving the by-passing of the respiratory chain - is ruled out.

The synthesis of vitamin $\mathrm{K} 2$ was also observed when menadione was added to the growth medium of other small-colony mutants of $S$. aureus; shikimic acid was not active. Thin-layer chromatography of the lipidic extract of such mutants (SHSL2, 3 and 4), grown in the presence of menadione, showed the characteristic spot for vitamin $\mathrm{K} 2$, while the same mutant grown in the absence of menadione lacked menaquinones. These results suggest that the mutants stimulated by menadione represent true vitamin $\mathrm{K}$-deficient mutants which will be of great interest in elucidating the unknown steps in the biosynthesis of menaquinones.

This investigation was supported by a grant from the Medical Research Council of Canada.

Laboratory of Bacterial Genetics,

A. SASARMAN*

M. Surdeanu

Institute Dr I. Cantacuzino,

Bucharest 35, Romania.

Institute of Microbiology and Hygiene,

Ville de Laval,

$P Q$, Canada.

V. Portilance

\section{Evidence for a Near Limiting Intracellular Concentration of a Regulator Substance}

In the course of evolution, if maximum metabolic economy is to be ensured, the level of expression of any gene becomes adjusted wherever possible so that the concentration of gene product will be sufficient, but not excessive. In the case of genes, the products of which have a regulatory role and are therefore only needed in small quantities, it may be difficult to prevent an excess of the regulator substance from being produced. Thus in the Escherichia coli lac system, although the intracellular concentration of repressor is undoubtedly low ${ }^{1}$, one $i$ gene produces sufficient repressor to control several sets of structural genes ${ }^{2}$. But I shall present here evidence to indicate that the regulator gene for the nitrate assimilation system in Aspergillus nidulans is produced in near limiting quantities such that one regulator gene makes sufficient product to control one set of structural genes, but insufficient to control two.

In Aspergillus nidulans, the nir gene specifies a regulator product which is essential for the expression of the structural genes which direct the synthesis of the enzymes nitrate reductase and nitrite reductase $e^{3,4}$. Mutation in the nir gene can lead to constitutive syntheses of these enzymes or to non-inducibility of these syntheses. In diploids between the non-inducible $\left(\right.$ nir $\left.^{-*}\right)$ and wild type $\left(\right.$ nir $\left.^{+}\right)$strains, these syntheses are inducible. Diploids between constitutive $\left(\right.$ nir $\left.^{c}\right)$ and nir ${ }^{+}$strains have a phenotype intermediate between those of the two components. To explain these dominance relationships and in particular the semi-dominance of the $\mathrm{nir}^{\mathrm{c}}$ allele, Pateman and $\mathrm{I}^{3}$ proposed that the nir gene product was not only essential for the expression of the structural genes in the presence of nitrate, but also repressed their activity in its absence. Results I shall present here are inconsistent with this explanation. Instead I propose that the nir product has only a positive regulatory role, and that the semidominance of the $n i r^{\circ}$ allele is a consequence of a dosage effect of the nir gene, indicating that the intracellular concentration of the nir product must be near limiting.

To distinguish between these two hypotheses it is necessary to measure the enzyme levels in various diploids. According to the original hypothesis the enzyme levels in diploids should show the following rankings

$$
\frac{n i r^{0}}{n i r^{0}}(\text { uninduced })=\frac{n i r^{\mathrm{c}}}{n i r^{-}}(\text {uninduced })>\frac{n i r^{\circ}}{n i r^{+}}(\text {uninduced })
$$

and

$$
\frac{n i r^{+}}{n i r^{+}}(\text {induced })=\frac{n i r^{+}}{n i r^{-}}(\text {induced })
$$

On a dosage effect hypothesis, however. these rankings would become

$$
\frac{n i r^{c}}{n i r^{\circ}}(\text { uninduced })>\frac{n i r^{c}}{n i r^{+}}(\text {uninduced })=\frac{n i r^{c}}{n i r}(\text { uninduced })
$$

and

\section{R. DOBARDZIC}

S. SONEA

Department of Microbiology and Immunology,

University of Montreal,

Montreal, PQ, Canada.

Received June 10; revised August 19, 1969.

* Now a visiting scientist in the Department of Microbiology and Immunology, University of Montreal, Canada.

\author{
Hayes, W., The Genetics of Bacteria and their Viruses (John Wiley, New \\ asarman, S., Surdeanu, M., Szabados, J., Creceanu, V., and Horodni- \\ ceanu, T., Rev. Canad. Bial., 27, 333 (1968).
Brodie, A. F., and Watanabe, T., Vitamins and Hormones, 24, 44 7 (1966).
}

